\title{
PROPOSAL OF WALKING METHOD TO AVOID FALLING DOWN USING VIBRATION ON LOOSE GROUND WITH SLOPE
}

\author{
Tomohiro WATANABE and Kojiro IIZUKA \\ Graduate school of Engineering and Science, Shibaura Institute of Technology, \\ Hukasaku, Minuma-ku, Saitama City, 337-8570, Japan \\ E-mail: nb19109@shibaura-it.ac.jp, iizuka@shibaura-it.ac.jp
}

\begin{abstract}
In this paper, walking method using vibration for leg typed rover is proposed to avoid falling down. Proposed method increases the tumble stability margin using vibration to improve stability during slope walking. In previous study, we confirmed that sinkage of the leg to the ground is increased when giving vibration. In the proposed method, posture of the leg typed rover is maintained to be stability using this feature. Experiments using a testbed was carried out. Moreover, we could get knowledge that the method using vibration was effective to avoid falling down the rover.
\end{abstract}

\section{Introduction}

The rovers which are exploration robots moving on the ground work in the space exploration missions. They achieved significant results in these missions ${ }^{12}$. The rovers which were used in these exploration missions are vehicle typed robots with wheels ${ }^{3}$. However, these rovers have the problem of low movement performance on the rough terrain and the loose ground ${ }^{4}$. Some rovers with moving mechanism except wheels are developed in order to improve the movement performance. In recent years, the rovers with leg mechanism are focused on as an exploration rover with high movement performance ${ }^{567}$.

The ground of Mars and the moon is covered with loose soil called regolith. Moreover, slope such as crater and Lunar hole exists on these grounds. Therefore, ability to climb loose ground with slope is important for the exploring rover. However, the surface of the loose ground is easy to destroy at motion of its legs. So, it is difficult to walk while maintaining posture stability. In this paper, walking method for the leg typed rover is proposed on a sloped terrains based on tumble stability margin. Proposed method increases tumble stability margin using vibration to avoid falling down during walking on a slope. In previous study, we confirmed that sinkage of the leg to the ground is increased when giving vibration $^{89}$. In the proposed method, posture of the leg typed rover is maintained to be stability using this feature. Experiments using a testbed was carried out. Moreover, we could get knowledge that the method using vibration was effective to avoid falling down the rover.

\section{Tumble stability margin}

Proposed walking method is based on tumble stability margin. Therefore, tumble stability margin is explained in this section.

In tumble stability margin, the moment in shaft passing through these two support points is calculated for evaluating stability of the leg typed robot. First, the reaction force $\bar{F}$ and moment $\bar{M}$ are obtained from the ground surface are calculated. The forces acting on the leg typed robot are gravity, reaction forces from the ground surface, and other external forces. $F_{G}$ and $M_{G}$ are a force and moment totaling the gravity acting on each link. $F_{a}$ and $M_{a}$ are force and moment for acceleration and deceleration of all link of the robot. Also, $F_{0}$ and $M_{0}$ are a force and moment totaling the other external forces except the contact force from the ground. The reaction force $\bar{F}$ and moment $\bar{M}$ are obtained from the ground 
surface can be expressed as follows Eq. (1) and Eq. (2).

$$
\begin{gathered}
\bar{F}=F_{a}-F_{G}-F_{0} \\
\bar{M}=M_{a}-M_{G}-M_{0}
\end{gathered}
$$

The ground touching point is defined as $P_{a}, P_{b}, \cdots, P_{n}$, and the position vector to the ground touching point is defined as $p_{1}, p_{2}, \cdots, p_{n}$, as shown in Figure 1 . The moment $M_{a b}$ around the line segment connecting two arbitrary ground touching points $P a, P b$ can be calculated from $\bar{F}$ and $\bar{M} . \bar{F}$ is the translational force working on the origin of the coordinate system. The moment $M_{b \bar{F}}$ around the ground touching point $P_{b}$, which is given by this translational force, is calculated from Eq. (3).

$$
M_{b \bar{F}}=\bar{F} \times P_{b}
$$

The value of this moment around the line segment connecting the points touching the ground $P_{a}, P_{b}$ is calculated from Eq. (4).

$$
M_{a b \bar{F}}=M_{b \bar{F}} \cdot \frac{\left(P_{a}-P_{b}\right)}{\left|P_{a}-P_{b}\right|}
$$

The value of this moment around the line segment connecting the points touching the ground $P_{a}, P_{b}$ is calculated from Eq. (5).

$$
M_{a b \bar{M}}=\bar{M} \cdot \frac{\left(P_{a}-P_{b}\right)}{\left|P_{a}-P_{b}\right|}
$$

The moment $M_{a b}$ around the line segment connecting two arbitrary points touching the ground $P_{a}, P_{b}$ is calculated from Eq. (6).

$$
M_{a b}=\bar{M} \cdot \frac{\left(P_{a}-P_{b}\right)}{\left|P_{a}-P_{b}\right|}+\bar{F} \cdot \frac{\left(P_{a} \times P_{b}\right)}{\left|P_{a}-P_{b}\right|}
$$

It is assumed that the ground touching points $P_{a}, P_{b}$ have not lifted up and that the moment $M_{a b}$ is not zero when more than two points are touching the ground. $n$ is the number of legs, and calculate the moment $M_{a b}$ for all combinations of $a=1$ to $n, b=1$ to $n(a \neq b)$. When all the determined tumble moments are cancelled by the remaining support legs, the minimum absolute value of the moment $M_{a b}$ can be calculated. After calculating the moment $M_{a b}$ for all combinations, the minimum absolute value of the moment $M_{a b}$ is choised. Moreover, tumble stability is calculated using this moment $M_{a b}$. Therefore, the tumble stability is calculated as moment $M_{a b}$ is divided by the weight of the robot. Eq. (7) shows the tumble stability.

$$
T_{S M}=\frac{\min \left|M_{a b}\right|}{m g}
$$

If the tumble stability is not satisfied, the value of tumble stability margin is set to zero. 


\section{Proposal of a walking method to avoid falling down using vibration}

When the rover walks on a slope, a large load acts on the legs in a low position. Therefore, the legs in a low position are easy to sink on the loose ground. Moreover, body of the rover leans backwards on slope by sinking these legs to the ground. So, risk of falling down the rover on the loose ground is high. In this study, walking method using vibration for the leg typed rover is proposed on sloped terrains to avoid falling down the rover. Shear strength of the ground is decreased when vibration is given to the ground. The reason is that particles of the ground move when giving vibration to the ground. Sinkage of the leg to the ground is increased by decreasing shear strength of the ground. In the proposed walking method, the legs in a high position vibrate. In this motion, body of the robot leans forwards on a slope. Posture of the rover is maintained to be stability by this motion.

\subsection{Detail of proposed walking method}

Detail of proposed walking method is explained in this paragraph. Figure 2 and Figure 3 show sequence of proposed walking method.

Proposed walking method is used for the leg typed rover which has 6 legs and based on wave gait. Figure 2 shows timing to vibrate the leg. First, the leg raises and moves forward, as shown in Figure 2(a). Next, the leg moves down, as shown in Figure 2(b). Next, the leg is vibrating when the leg touches the ground, as shown in Figure 2(c). Moreover, leg sinks on the ground. Finally, vibration stops when the leg finish sinking on the ground, as shown in Figure 2(d).

Next, sequence of proposed walking method is explained. First, 4 legs in a high position vibrate, as shown in Figure 3(a). Moreover, body of the rover leans forwards on a slope. Next, A leg moves forward one by one, as shown from Figure 3(b) to Figure 3(d). When all legs finish moving, body of the rover moves forward, as shown in Figure 3(e). Moreover, 1 cycle of this gait is finished.

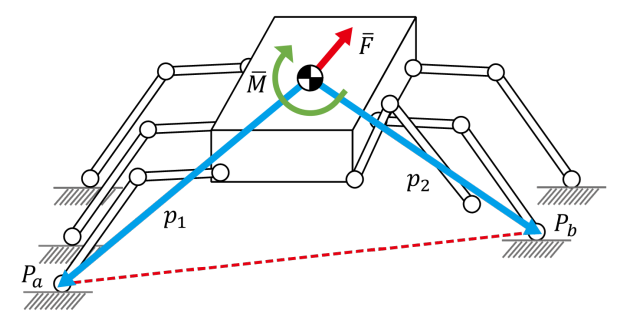

Fig. 1. Model of leg typed robot

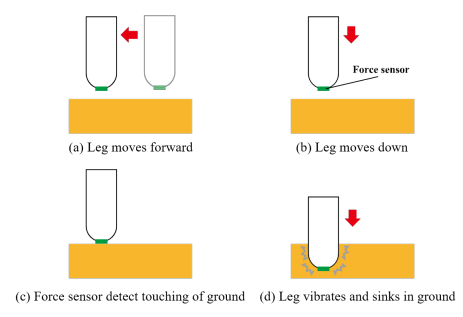

Fig. 2. Vibration timing when walking

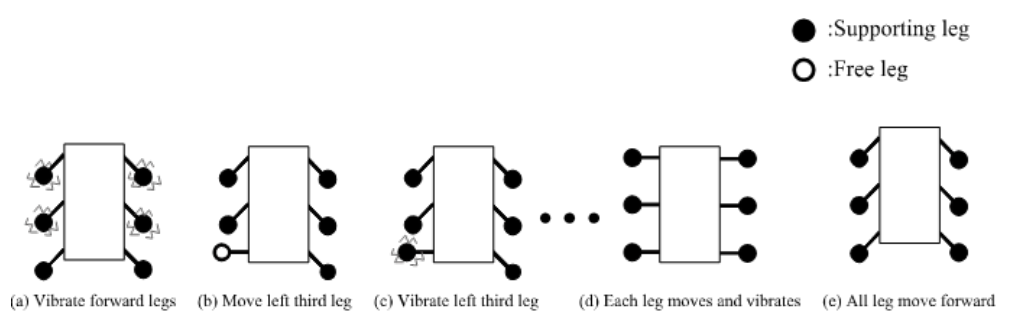

Fig. 3. Sequence of proposed walking 


\section{Slope walking experiment using testbed}

\subsection{Description of experiment contents}

The slope walking experiment is conducted using the testbed to confirm effectiveness of the proposed walking method. Detail of experimental method is explained in this paragraph. The testbed walks on the loose ground with slope. Kind of sand which consists in the ground is silica No.5. Detail of the testbed and evaluation content to be measured are explained in the following paragraph. Three different walking method are used. There walking methods are shown in Figure 4. In proposed walking, the motion that 4 legs in a high position vibrate (Figure $4(\mathrm{~A})(\mathrm{a})$ ) is operated. In this motion, vibration time is $10 \mathrm{~s}$. Next, the leg raises and moves forward, as shown in Figure 4(A)(b). The leg is vibrating when the leg is lowering, as shown in Figure 4(A)(c). In this motion, vibration time is 10s. Each leg moves amd vibrates. In next cycle, the motion which is shown in Figure $4(\mathrm{~A})(\mathrm{a})$ is operated when the motion which is shown in Figure 4(A)(e) is finished. Conventional walking was proposed in previous study $^{8}$. In conventional walking, the motion that 4 legs in a high position vibrate (Figure $4(\mathrm{~B})(\mathrm{a}))$ is operated at first only. In next cycle, the motion which is shown in Figure 4(B)(b) is operated when the motion which is shown in Figure $4(\mathrm{~B})(\mathrm{e})$ is finished. In no vibration, testbed does not operate motions that legs vibrate. Amount of sinking legs to the ground is $30 \mathrm{~mm}$ in the method of proposed walking and conventional walking. The slope of ground changes by $5^{\circ}$ between $0^{\circ}$ and $20^{\circ}$. The number of trials is 5 times. Figure 5 shows the environment of the slope walking experiment. The number of walking steps is 5 steps. Table 1 shows condition of this experiment.

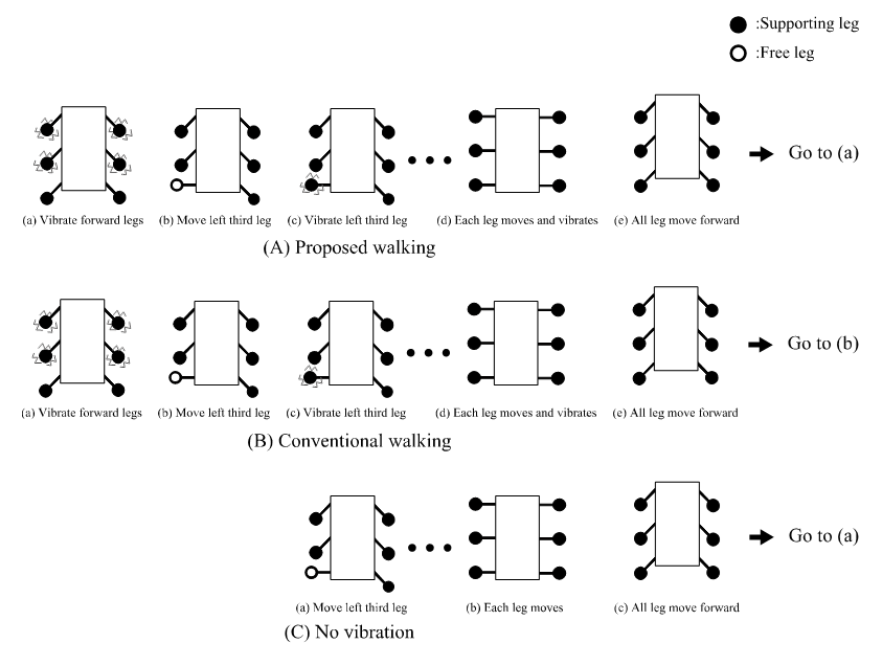

Fig. 4. Kinds of walking method using slope walking experiment

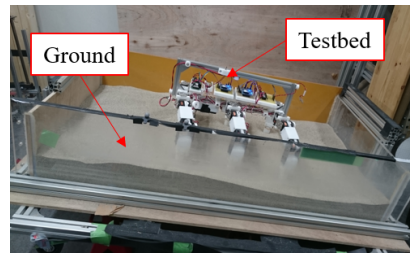

Fig. 5. Environment of experiment

\begin{tabular}{cc} 
Table 1. & Conditions of slope walking experiment \\
\hline Item & Condition (value) \\
\hline Slope angle $\left[^{\circ}\right.$ ] & $0,5,10,15,20$ \\
Number of trials & 5 \\
Number of walking cycle & 5 cycles \\
Kind of sand & Silica No.5 \\
\hline
\end{tabular}




\subsection{Description of measurement and evaluation contents}

In this experiment, tumble stability margin backwards on a slope is calculated as stability evaluation of posture. Risk of falling down the rover on the loose ground is high when the rover walks on the loose ground with a slope. Therefore, tumble stability margin backwards on a slope is focused on as stability evaluation of posture. Calculation of tumble stability margin backwards on a slope is explained and shown in Figure 6. When leg c is free leg, tumble stability margin is calculated by the moment around the line segment connecting point $b$ and point $f$, as shown in Figure 6(a). When leg $\mathrm{f}$ is free leg, tumble stability margin is calculated by the moment around the line segment connecting point $\mathrm{c}$ and point $\mathrm{e}$, as shown in Figure 6(b). When leg c and leg $\mathrm{f}$ are not free leg, tumble stability margin is calculated by the moment around the line segment connecting point $\mathrm{c}$ and point $\mathrm{f}$, as shown in Figure 6(c). The more tumble stability margin backwards on slope is large, the more posture of the rover is stability. In this experiment, the rover is not taken force except the contact force from the ground. Moreover, force and moment for acceleration and deceleration of all link of the rover are very small because the rover dose static walking. Therefore, the reaction force $\bar{F}$ is obtained from the ground surface is expressed by Eq. (1) in this experiment. Moreover, the moment $M_{a b}$ is calculated by Eq. (9).

$$
\begin{gathered}
\bar{F}=-F_{G} \\
M_{a b}=\bar{F} \cdot \frac{\left(P_{a} \times P_{b}\right)}{\left|P_{a}-P_{b}\right|}
\end{gathered}
$$

Posture of body is measured to confirm preventing from turning backwards on a slope by the proposed method. Figure 7 shows testbed posture to measure. Moreover, the walking amount of each walking method is measured to confirm reducing slip distance.

\subsection{Details of the testbed}

Table 2 shows specifications of the testbed. Moreover, overview and structure of the testbed are shown in Figure 8. The leg of testbed is consisted of three joints and does up down movement, forward and backward. The vibration motor is mounted in the leg. It is need judgment of contact between the ground and the legs and posture of the testbed to calculate tumble stability margin backwards on a slope. The force sensor (FSR400) is used to judge contact between the ground and the legs. This force sensor is connected top of the foot, as shown in Figure 9(a). Acceleration sensor (ADXL345) is used to measure posture of the testbed. Figure 9(b) shows measuring direction about posture of the testbed. Sampling frequency of these sensors is $2 \mathrm{~Hz}$.

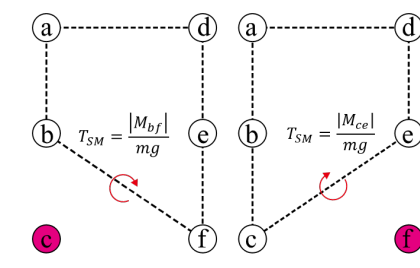

$\begin{array}{ll}\text { (a) Pattern that leg-c is free leg } & \text { (b) Pattern that leg-fis free leg }\end{array}$

Fig. 6. Caluculation method of tumble stability margine in down slope

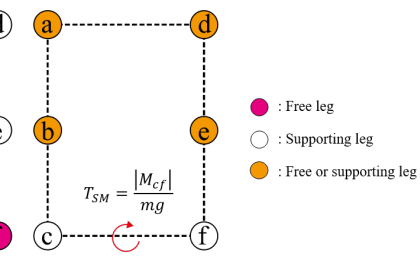

(c) Other patterns

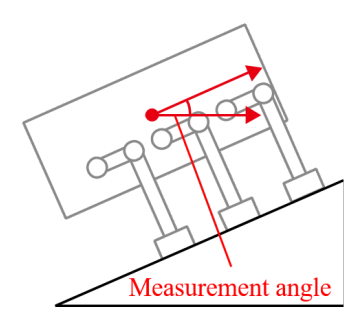

Fig. 7. Testbed posture to measure 
Table 2. Specifications of testbed

\begin{tabular}{cc}
\hline Item & Condition (value) \\
\hline Weight & $5.9 \mathrm{~kg}$ \\
Size & $\mathrm{H}: 163 \mathrm{~mm} \times \mathrm{W}: 422 \mathrm{~mm} \times \mathrm{L}: 400 \mathrm{~mm}$ \\
Servo motor & KRS6003R2HV ICS \\
Vibration motor & TP-2528C-24 \\
Softwear & Visual Studio 2019 \\
\hline
\end{tabular}
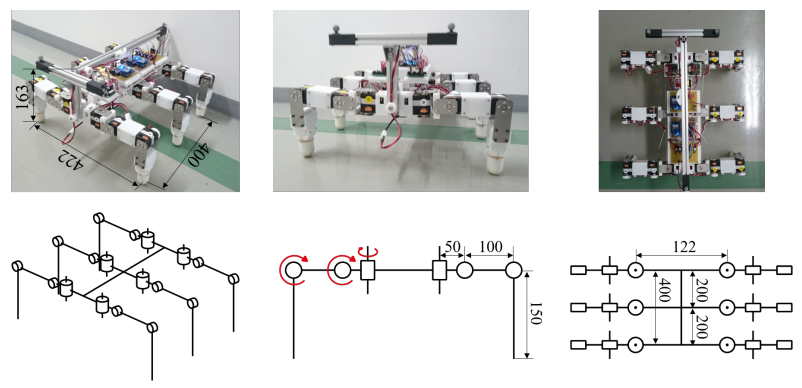

Fig. 8. Overview and structure of leg typed testbed

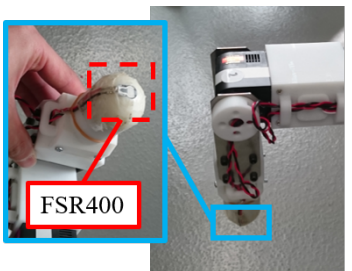

(a) Placement of force sensor

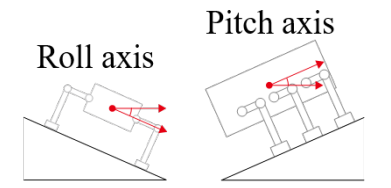

(b) Measurement angle by acceleration sensor

Fig. 9. Sensing system of leg typed testbed

\subsection{Results and Discussion}

Figure 10 shows graph comparing walking distance of the testbed. The testbed using no vibration was fallen down on the loose ground with slope of $15^{\circ}$ and $20^{\circ}$. Moreover, the testbed using conventional walking was fallen down on the slope of $20^{\circ}$. The testbed using proposed walking avoided falling down when walking on the slope of $20^{\circ}$. Each amount of movement using proposed walking and conventional walking is longer than that using no vibration. In slope of $15^{\circ}$, amount of movement using proposed walking is longer than that using conventional walking. Therefore, proposed walking is more effective than other walking methods.

Figure 11 shows the graph of tumble stability margin backwards in a slope of $10^{\circ}$. Figure 12 shows the graph of posture of the testbed in a slope of $10^{\circ}$. In Figure 11, amount of tumble stability margin using proposed walking and conventional walking are almost same. Amount of tumble stability margin using no vibration is smaller than that using other walking method. In Figure 12, it is confirmed that posture of the testbed is increased when the testbed walked using no vibration. Figure 13 shows the graph of tumble stability margin backwards in a slope of $15^{\circ}$. Figure 14 shows the graph of posture of the testbed in slope of 
$15^{\circ}$. In Figure 13, amount of tumble stability margin using conventional walking is smaller than that using Proposed walking. In Figure 14, posture of the testbed using conventional walking is larger than that using proposed walking. Therefore, it was confirmed that tumble stability margin and posture of the testbed is increased using proposed walking. Figure 15 shows the graph of tumble stability margin backwards on slope and posture of the testbed in a slope of $20^{\circ}$. In slope of $20^{\circ}$, posture of the testbed is increased when the testbed walked using proposed walking. Tumble stability margin is zero but the testbed avoided falling down when walking on the loose ground. The reason that testbed avoided falling down is to sink the legs in the ground. Resistance which avoid testbed falling down is caused because friction between the leg and the ground is caused by sinking the legs in the ground. Figure 16 shows this resistance force. In this experiment, this resistance does not include in the formula about tumble stability margin. Therefore, tumble stability margin which includes this resistance will be proposed in future study.

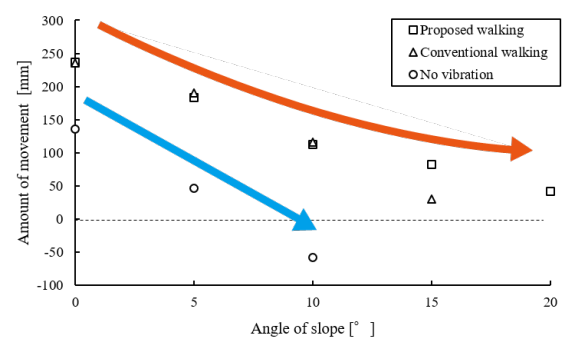

Fig. 10. Amount of movement in each walking

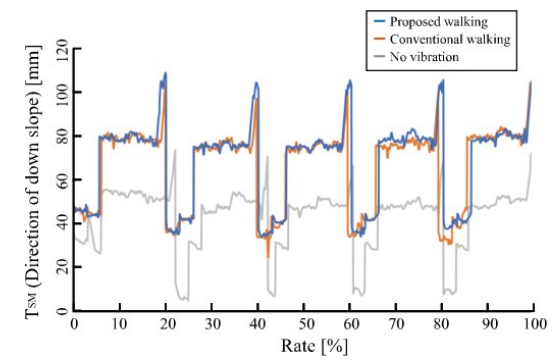

Fig. 11. Tumble stability margin on slope with $10^{\circ}$

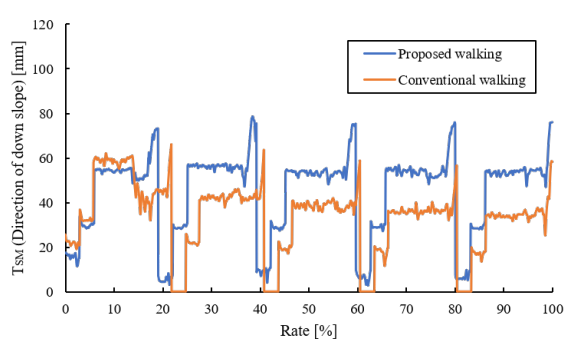

Fig. 13. Tumble stability margin on slope with $15^{\circ}$

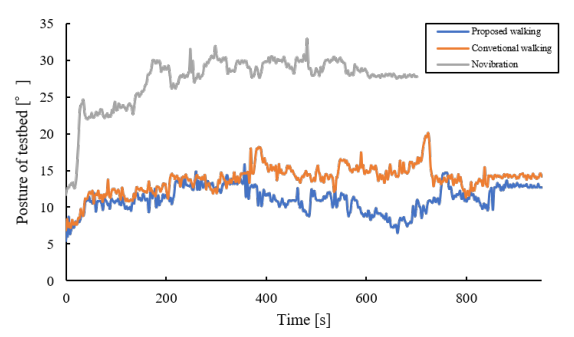

Fig. 12. Posture of testbed on slope with $10^{\circ}$

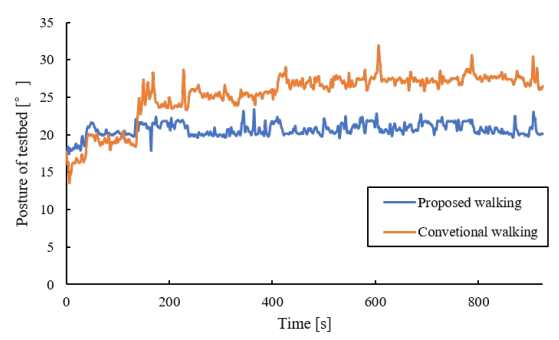

Fig. 14. Posture of testbed on slope with $15^{\circ}$ 


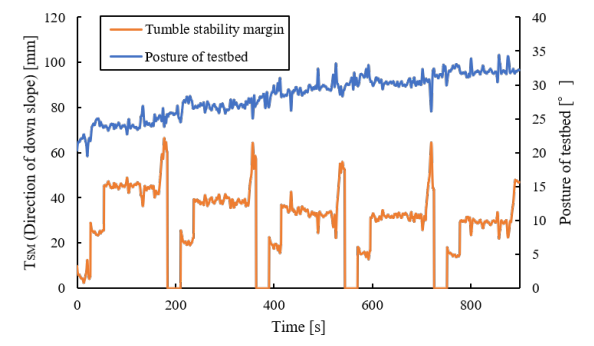

Fig. 15. Tumble stability margin and posture of testbed on slope with $20^{\circ}$

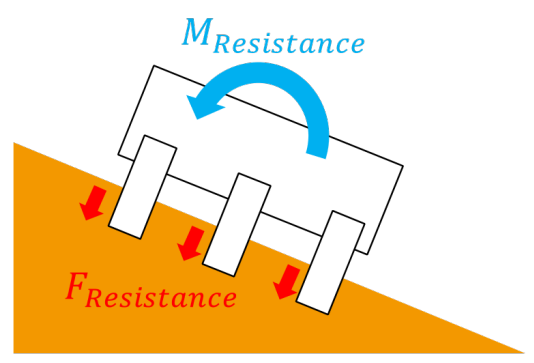

Fig. 16. Resistance force and moment by sinkage

\section{Conclusion}

In this paper, walking method which can avoid falling for the leg typed rover was proposed on the loose ground. Proposed method is used feature that sinkage of the leg to the ground is increased when giving vibration. To validate the proposed method, experiments using a testbed was carried out. The result are shown that the tumble stability margin was better when using proposed method on loose ground with slope. From these experimental results, we could get knowledge that the method using vibration was effective to avoid falling down the rover.

It is considered that the resistance which avoids testbed falling down is caused by sinking the legs in the ground. In future study, tumble stability margin which includes this resistance will be proposed.

\section{Acknowledgements}

This work was supported by JKA and its promotion founds from KEIRIN RACE.

\section{References}

1. Chhaniyara, S., Brunskill, C., Yeomans, B., Matthews, M.C., Saaj, C., Ransom, S. and Richter, L., Terrain trafficability analysis and soil mechanical property identification for planetary rovers: A survey, Journal of Terramechanics, Vol.49 (2012), pp.115-128.

2. Ueno, H., State of art for planetary robotics on space agencies, Journal of the Robotics Society of Japan, Vol. 32, No. 5 (2014), pp.431-434 (in Japanese).

3. NASA, JPL, California institute of technology pasadena, Mars pathfinder, NASA Facts, available from 〈https://www.jpl.nasa.gov/news/fact_sheets/mpf.pdf $\rangle$, (accessed on 6 November, 2019).

4. Kubota, T., Rover design for lunar of planetary exploration, Vol. 17, No. 5 (1999), pp.609--614 (in Japanese).

5. Spenneberg, D., Kirchner, F. and de Gea, J., Ambulating robots for exploration in rough terrain on future extraterrestrial missions, Proceedings of 7th International Symposium on Artificial Intelligence Robotics and Automation in Space (2004).

6. Komizunai, S., Konno, A., Abiko, S. and Uchiyama, M., Development of a static sinkage model for a biped robot on loose soil, 2010 IEEE/SICE International Symposium on System Integration, (2010).

7. Yeomans, B. and Saaj, C. M., Towards terrain interaction prediction for bioinspired planetary exploration rovers, Bioinspiration \& Biomimetics, Vol. 9, No. 1 (2014), pp.1-15.

8. Watanabe, T. and Iizuka, K., Study on legged typed rovers with function of vibration/stop to travel on loose soil, 15th ISTVS European-African Regional Conference, (2019).

9. Watanabe, T. and Iizuka, K., Proposal of walking to reduce slipping behavior using compaction effect of loose soil caused by a propagation of vibration for small light lunar planetary exploration rovers with legs, The Japan Society of Mechanical Engineers, (2019).Vol. 86, No. 886 (2020), (in Japanese). 